\title{
Research of Blockchain Financial Application Standard: Multi-dimensional Perspective and Development Path
}

\author{
Xidi Zhang ${ }^{1 *}$ \\ 1. School of Finance, Zhejiang University of Finance and Economics, Hangzhou 310018, China \\ *Corresponding author Email: 1848099854@qq.com
}

\begin{abstract}
Standards are the cornerstone of the development of blockchain financial applications.For a better promotion, we need to fully push forward the design of the whole framework of relevant standard systems, including basic standards, business and application standards, process and method standards, trust and interoperability standards, information security standards, etc.We should accurately grasp the core contents, standard research requirement for instance, of blockchain financial application, which contains consensus protocol, smart contract, data format and IT Operations Management.In order to realize the vertical and deepening goal of China's blockchain finance application standard research, this paper proposes the development path of the research of blockchain finance application standard, including: vigorously promoting the large-scale application and avoiding the misunderstanding effect of blockchain finance and breaking the talent bottleneck.We should also seize the development heights and strive for more international voice of blockchain financial application standards.
\end{abstract}

Keywords: Blockchain, Financial application, Standard research, Multi-dimensional perspective, Development path.

\section{INTRODUCTION}

Blockchain is a shared database characterized by decentralization, openness, independence, security and anonymity. Its characteristics of unfordable, whole-process traces, easy traceability, transparency and collective maintenance lay a solid foundation for trust and a reliable cooperation mechanism, which brought broad innovation space for the business model and social reform and caused widespread concern and high attention of the society. The standard is discourse power. China needs to intensify the practice of the blockchain financial industry, deepen the multi-dimensional perspective and vigorously promote the development of the blockchain financial application standard.

\section{THE WHOLE FRAMEWORK DESIGN OF BLOCKCHAIN FINANCIAL APPLICATION STANDARD SYSTEM}

The blockchain financial application standard system can be divided into five categories: basic standards, business and application standards, process and method standards, trust and interoperability standards, information security standards.

\subsection{Basic Standards}

The basic standards of blockchain financial application refer to the criteria that are widely used as the basis of other standards and have broad guiding significance. The standards often make unified provisions on the common factors of blockchain financial application, such as concepts, number systems and general rules.[1] For example, the standards specify the technical general principles basic standards like revised regulations, requirements and documentation rules, and the language general principles basic standards like terms, symbols, codes, and cartographic convention.

\subsection{Business and application standards}

The business and application standards are the standards formulated by coordinated and unified work matters. With a wide range of applications, blockchain 
finance can be connected with all walks of life and has a wide range of applications. The application scenarios in real life are developing exploitously. Therefore, it is necessary to make clear provisions on the scope, responsibilities, rights, procedures, requirements and effects involved in blockchain finance, so as to better coordinate and uniformly promote the development and implementation of blockchain financial application business.[2]

\subsection{Process and method standards}

Blockchain financial application, as a complex new financial technology innovation, requires a large number of process and method standards in the adoption process to guide the developers to work better together.The process and method standards refer to the criteria that ensure suitability by specifying processes and methods.It will affect the availability of blockchain financial application projects and the efficiency of the operational process, simplify development communication, optimally advance multiple scene implementation.It also relates to environmental protection and so on.

\subsection{Trust and interoperability standards}

The trust and interoperability standards are the system stability and capability level standards.Trust, including reliability, maintainability and availability, is very important for blockchain financial applications.Only when the system reaches a certain level of credibility can it ensure the safe development of financial service.Failure or malfunction of the system due to unreliability can be catastrophic.At the same time, to realize efficient digital exchange and coordination of relevant information, blockchain financial application projects must comply with interoperability requirements, so as to further achieve a common development goal.

\subsection{Information security standards}

Blockchain financial application information security involves computer security operating system, various security protocols, security mechanisms (digital signature, message authentication, data encryption, etc.).The information security standards are standards related to the information security status of blockchain.It makes strict specifications for information confidentiality, authenticity and integrity, including information security management implementation rules and system specifications. The rules serve as basic guiding documents to guide developers to implement and maintain information security within the project team. The specifications are detailed requirements for establishing, implementing and maintaining information security regulation systems for blockchain projects.

\section{THE CORE CONTENTS OF THE DEVELOPMENT OF BLOCKCHAIN FINANCIAL APPLICATION STANDARDS}

\subsection{Consensus protocol}

The existence of consensus protocol enables each node, concerned with maximizing its own interests, to voluntarily and honestly abide by the pre-set rules of the agreement in a distrustful market.It faithfully records every transaction data, containing work proof mechanism, equity proof mechanism, share authorization proof mechanism and the verification pooling of POOL.[3] The requirements for consensus protocol standards development for blockchain financial applications are as follows: first, security requirements, namely whether it can prevent attacks such as secondary payment and selfish mining, and whether it has good fault tolerance. Secondly, scalability requirements, that is, whether network node expansion is supported. The third is the high performance efficiency requirement, which is the time delay between the transaction consensus being recorded in the blockchain and final confirmation, and the number of confirmed transactions that the system can process per second. The fourth is low energy consumption requirements, that is, in the process of reaching a consensus, the amount of computing resources consumed by the system, including CPU, memory, etc.

\subsection{Smart Contract}

Smart contract is computer protocol designed to propagate, validate or enforce contracts in an information-based manner, which effectively reduces other transaction costs. In the blockchain finance application project, smart contracts execute computerized trading protocols of treaty articles, allowing trusted, traceable and irreversible transactions to take place without a third party. The smart contract standard development for blockchain finance applications requires the normative transformation of financial transactions into contract codes rigorously audited by trusted third parties. The control of value stream of blockchain system is realized through contract code.By virtue of the distributed characteristics of blockchain, authoritative information can be issued through the management of different nodes, making financial business observable, verifiable, regulated and unchangeable, so as to improve the efficiency and credibility of financial business.

\subsection{Data Format}

Data format describes the rules for storing data in files or records. It can be text format in character form that occupies more storage space but high transparency, or compressed format in binary data form that takes up 
less storage space but lacks transparency. Commonly used blockchain financial application data can be divided into simple types and compound types. It can also be divided into array type, record type, collection type, and file type according to different constructors. Requirements for data format standard development for blockchain financial applications are as follows: The first is to ensure that all the information needed is recorded. Secondly, improve storage efficiency to ensure full utilization of storage space. The third is the standardization of format to ensure the exchange of data between relevant data processing systems.[4]

\subsection{IT Operations Management}

IT Operations Management helps financial institutions establish IT Operation and maintenance modes that respond quickly and adapt to the blockchain business environment and business development.It helps establish process framework based on information technology infrastructure library, practice operation and maintenance automation goals.Requirements for development of operation and maintenance management standards are as follows: First of all, establish business value as the core, business driven oriented IT governance and management thought, jointly serve financial institutions to achieve strategic goals. The second is to establish the key business service model that is compatible with the future development vision, IT architecture and management mode of financial institutions, and realize the automation of key business processes. Thirdly, integrate the network monitoring, system monitoring and database monitoring tools built by financial institutions into a single point of management platform to achieve fast fault location and efficient information sharing. It also helps conduct rapid root cause analysis for financial institutions in business models to improve the speed and accuracy of problem solving.

\section{BOOST THE VERTICAL AND DEEPENING DEVELOPMENT OF BLOCKCHAIN FINANCIAL APPLICATION STANDARDS}

\subsection{Actively participate in the development of international standards}

International standards refer to thestandards formulated by the International Organization for Standardization (ISO), the International Electrotechnical Commission (IEC), and the International Telecommunication Union (ITU), as well as those formulated by other international organizations confirmed and published by the ISO and used uniformly throughout the world.Participating in the development of international standards for blockchain financial application can more effectively promote the blockchain industry development based on China's national conditions, improve China's blockchain financial application standard system, and achieve the application of blockchain finance to reach or even exceed the world's advanced level.

\subsection{Vigorously promote national standards development}

National standards of the People's Republic of China, referred to as GB, have a very high legal status. The mandatory standard of the national standard for blockchain financial applications is titled with "GB" and the recommended standard is titled with "GB/T".By the middle of 2021, there are three standards to be included in the national standard project of blockchain financial application, namely, blockchain and distributed ledger technology reference architecture, smart contract implementation specification for blockchain and distributed accounting technology, the guide to the application of blockchain and distributed ledger technology.

\subsection{Timely release of industry standards}

Industry standards are formulated by the centralized industrial department standards to unify the technical requirements of a certain industry in the whole country. In the context of the booming blockchain financial application industry in China, it is urgent to establish a unified industry standard. The industry standards should not only give full play to the technical advantages of blockchain, but also organically connect the characteristics of blockchain financial application in different fields. The standards of each system shall be coordinated and unified, and shall not be duplicated or in conflict with relevant national standards. The development of industry standards for blockchain financial applications is of great significance and must be highly concerned and actively promoted. In this process, the development of industry standards for blockchain financial application should have a high degree of risk prevention and control awareness, but also prevent timid and missed opportunities.

\subsection{Strongly encourage local standards}

Local standards for blockchain financial application are approved and issued by local (province, autonomous region, municipality directly under the Central Government) standardization authorities or professional competent authorities, and implemented uniformly in a certain region. Formulating local standards for blockchain financial application is in line with China's reality which have huge differences in economic and technological environments because of the geographical vastness. Establishing local standards is conducive to 
promoting the development and application of advanced technical standards by financial institutions and improving the quality of blockchain financial application products and services. It helps guide the development of blockchain financial application technology, strengthen industry and social supervision, and better safeguard social interests and consumer rights and interests. It can also better realize the standardized information exchange and sharing, and improve the public welfare services.

\subsection{Fully open group standards}

Group refers to social organization that has legal person status and corresponding professional technical, standardized and organizational management capabilities such as institute, association, chamber of commerce, federation and industrial technology alliance. Blockchain financial application group standards are standards independently formulated and published in accordance with standard development procedures by groups and adopted voluntarily by the society. Since 2020, blockchain financial application community standards have maintained a rapid momentum of development. In Guangdong, Zhejiang, Shanghai and other regions with mature blockchain industry, the development of group standards for blockchain financial application is more active. The content focuses on the combination and design of blockchain and specific application scenarios in the financial field.

\section{DEVELOPMENT PATH OF BLOCKCHAIN FINANCIAL APPLICATION STANDARD RESEARCH}

\subsection{Promote the large-scale application of blockchain finance}

Vigorously promote the development of blockchain financial application projects, and actively promote large-scale applications on the premise of avoiding systemic risks. Combined with the successful experience of the digital currency project of the People's Bank of China, it will accelerate the maturity scenarios and provide industrial soil for the development of blockchain financial application standards.

\subsection{Avoid blockchain financial misunderstanding effect}

We should carefully identify industry risks and effectively prevent and control financial systemic risks in the process of blockchain application. Avoid the pitfalls through learning and communication, and strive to develop a good degree of perfection, strong applicability and good universality.

\subsection{Break the talent bottleneck}

Set up blockchain undergraduate major, and list blockchain industry base. Actively cultivate talents in the field of blockchain, especially senior talents in the development of blockchain financial application standards, strengthen international industry exchanges and continue to promote standardization research at home and abroad.[5]

\section{CONCLUSION}

China has made a certain amount of accumulation in the development of blockchain financial application standards, not only reflected in the establishment of the standard system framework including the relevant basic standards, business and application standards, process and method standards, credibility and interoperability standards, information security standards.Moreover, positive exploration and practice such as consensus protocol, smart contract, data format and IT operations management have been made in the core content of blockchain financial application standardand yielded relatively gratifying results. We should actively participate and promote in domestic and foreign standard development.We should also timely promulgate industry standards, strongly encourage local standards, roundly open group standards.Moreover, the goal of avoiding the error effect can be achieved through the large-scale application of blockchain finance.We also need to break through the talent bottleneck and other development path issues, seize the development heights and strive for more international voice of blockchain financial application standards.

\section{REFERENCES}

[1] Shubing Shan, Bingbing $\mathrm{Hu}, \mathrm{Xiao}$ Chen, Comparative Analysis and Consideration of Domestic and foreign blockchain financial Standards, Proceedings of the 17th China Standardization Forum, November 2020, p112-115.

[2] Li Xin, How to View the Release of the first Blockchain standard in the Financial Industry, China Urban and Rural Finance News, March 16, 2020, edition A02.

[3] Yang Jingli, Zhang Ruiyang, Blockchain Application Acceleration calls for Industry Standard Innovation, Standardization in China, 2019 (1), P6-13.

[4] Lei Zhao, How to Regulate blockchain: Application Scenarios and Technical Standards, China Law Review, 2018 (6), p177-185.

[5] Yun Liu, Order of Standards and disorder of blockchain, Mass Standardization, 2018 (2), P5. 